The Caldicott report

\section{The Caldicott report and patient confidentiality}

\section{A Crook}

\section{An introduction for the pathologist}

n 1997, the Caldicott committee presented its report on patient confidentiality. ${ }^{1}$ The impetus behind this were concerns about patient information and security. ${ }^{23}$ For example, there had been reports in the press that patient hospital records could be freely accessed and that patient notes had ended up lying around in village streets for all and sundry to read.

The committee came up with six main principles as follows.

(1) One should justify the purpose of holding patient information.

(2) Information on patients should only be held if absolutely necessary.

(3) Use only the minimum of information that is required.
(4) Information access should be on a strict need to know basis.

(5) Everyone in the organisation should be aware of their responsibilities.

(6) The organisation should understand and comply with the law.

National Health Service (NHS) organisations should have Caldicott guardians who have responsibilities to safeguard and govern the use of patient information. The guardian is usually a board level health professional or their deputy. They should develop local protocols for information disclosure, restrict access to patient information by enforcing strict need to know principles, and regularly review and justify the uses of patient information.

Recommendation 1. Every dataflow, current or proposed, should be tested against basic principles of good practice. Continuing flows should be tested regularly

Recommendation 2. A programme of work should be established to reinforce awareness of confidentiality and information security requirements among staff within the National Health Service (NHS)

Recommendation 3. A senior person, preferably a health care professional, should be nominated in each health organisation to act as guardian, responsible for safeguarding the confidentiality of patient information

Recommendation 4. Clear guidance should be provided for those individuals/bodies responsible for approving uses of patient identifiable information

Recommendation 5. Protocols should be developed to protect the exchange of patient identifiable information between NHS and non-NHS bodies

Recommendation 6. The identity of those responsible for monitoring the sharing and transfer of information within local protocols should be clearly communicated

Recommendation 7. An accreditation system that recognises those organisations following good practice with respect to confidentiality should be considered

Recommendation 8. The NHS number should replace other identifiers wherever practicable taking account of the consequences of errors and particular requirements for other specific identities

Recommendation 9. Strict protocols should define who is authorised to gain access to patient identity where the NHS number or other coded identifier is used

Recommendation 10. Where particularly sensitive information is transferred, privacy enhancing technologies (such as encrypting identifiers or patient identifying information) must be explored

Recommendation 11. Those involved in developing health information systems should ensure that the best practice principles are incorporated during design stage

Recommendation 12. Where practicable the internal structure and administration of databases holding patient identifiable information should reflect the principles developed inthis report

Recommendation 13. The NHS number should replace the patient's name on items of service claims made by genera practitioners as soon as practically possible

Recommendation 14. The design of new systems for the transfer of prescription data should incorporate the principles developed in this report

Recommendation 15. Future negotiations on pay and conditions for general practitioners should where possible avoid systems of payment that require patient identifying details to be transmitted

Recommendation 16. Consideration should be given to procedures for general practice claims and payments that do no require identifying information to be transferred, which can then be piloted

Figure 1 Recommendations of the Caldicott committee to ensure patient confidentiality.

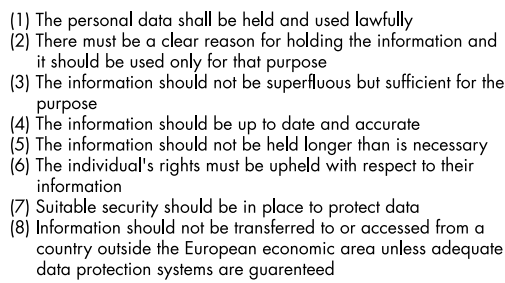

Figure 2 The eight main principles of the Data Protection Act.

The Caldicott committee also came up with recommendations for ensuring patient confidentiality, which are summarised in fig 1 .

"National Health Service
organisations should have
Caldicott guardians who have
responsibilities to safeguard and
govern the use of patient
information"

These principles regarding patient confidentiality are also entrenched in the NHS core plan. Indeed, the NHS plan core principle 10 states that "patient confidentiality will be respected throughout the process of care". The Data Protection Act 1998 is also relevant in this context. The aim of this act is to uphold an individual's right to privacy with regard to the processing of personal data. There are eight main principles of this act (fig 2).

Where does this lead us as pathologists? First, I suspect that patient confidentiality will feature more and more within the NHS with the associated potential for litigation. Caldicott issues will probably be used as NHS performance indicators based partly upon the Caldicott audit returns. For example, we will need to ensure secure transmission and distribution of our patients' data, such as accurate faxing of laboratory results to information safe havens, and use password protected computer systems. Information technology security should comply with BS7799 and the Data Protection Act. In addition, we should take particular care of the safety of patient notes and ensure patient consent where necessary regarding confidentiality issues. The only time that patient information can be divulged to a third party is if the patient has given their properly informed consent for this to happen, or if the data are totally anonymised to prevent identification of the patient from the details given.

The General Medical Council statement on confidentiality (September 2000) also remarked that as doctors we hold information about patients, which is private and sensitive. This information must not be given to others 
unless the patient consents or the disclosure can be justified. We will also need to establish training programmes about patient confidentiality for our staff and help map patient information flows, to name but a few areas. Nonconsensual data sharing may be deemed contrary to medical ethics and where possible anonymised patient data should be used..$^{4-7}$

These aspects of patient confidentiality are summarised as the Caldicott audit points shown in table 1 .
Section 60 of the Health and Social Care Act, passed by parliament in May 2001, gives the secretary of state for health the power to allow the processing of patient information for medical purposes if these purposes are in the public interest (for example, cancer registries). "Patient information" in this context means any health or medical information about the patient, whether identifiable with an individual or not. The government also agreed to the establishment of a statutory advisory committee (the patient information advisory group) to keep the provisions on confidential data and their use by the secretary of state under review.

In summary, patient information and confidentiality issues will probably gain increasing importance in the following years within the NHS. A balance between individual data privacy and useful information exchange for the benefit of society will need to be struck. ${ }^{8}$ On that note, do not forget the Caldicott principles' mnemonic, a reminder of Dame Fiona Caldicott herself:

\section{Table 1 Caldicott audit points}

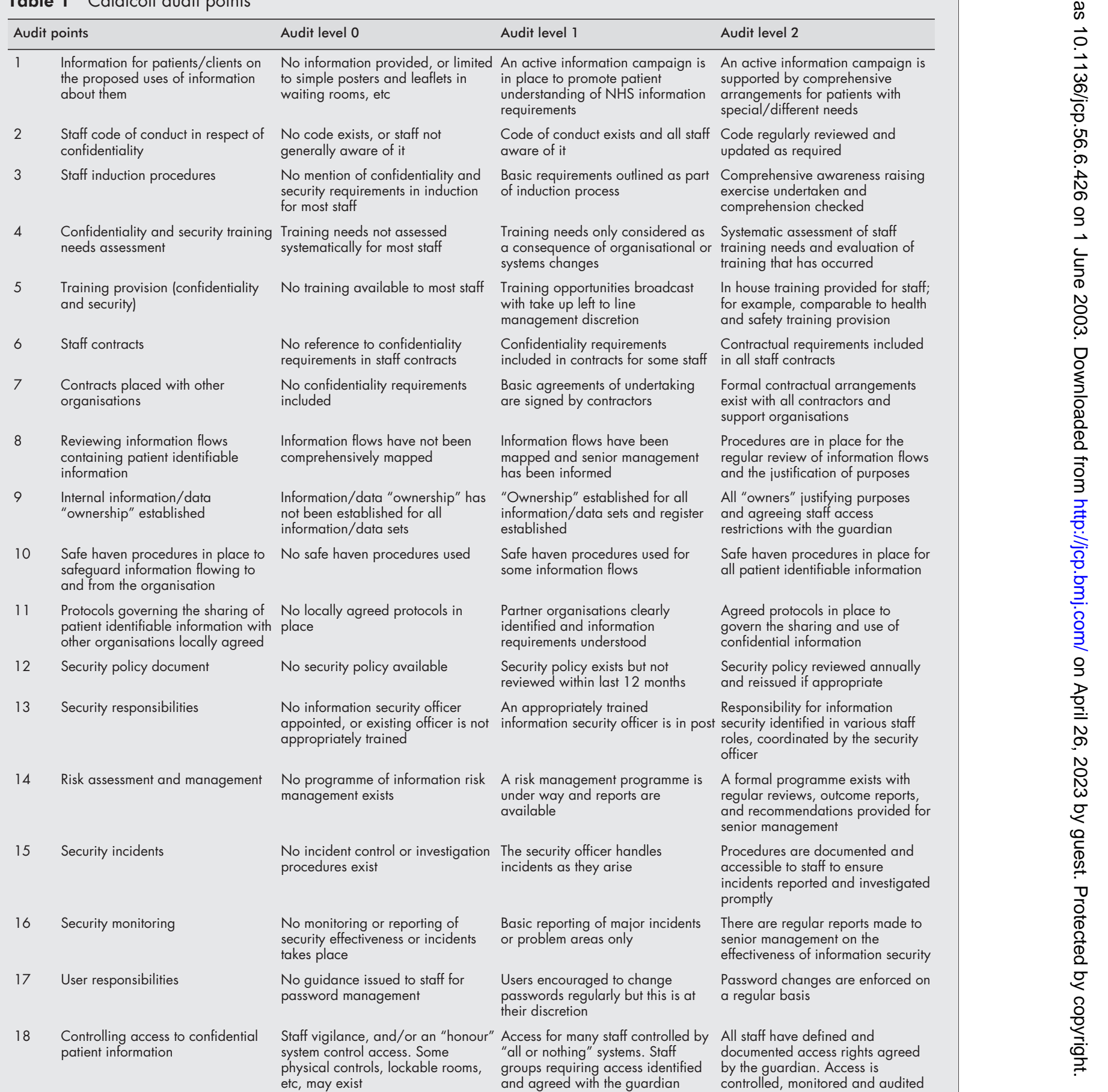




\section{FIONA C}

Formal justification of purpose.

Information transferred only when absolutely necessary.

Only the minimum required.

This may help us to focus on patient confidentiality in our clinical work; remembering it to be an important part of risk management and clinical governance.

J Clin Pathol 2003;56:426-428

Need to know access controls.

All to understand their responsibilities.

Comply with and understand the law.
Correspondence to: Dr M A Crook, Guy's, St Thomas's, and University Hospital Lewisham, London SE 13 6LH, UK; martin.crook@uhl.nhs.uk

\section{REFERENCES}

1 The Caldicott report. IHRIM 1999;40:17-19.

2 Wiederhold G. Future of security and privacy in medical information. Stud Health Technol Inform 2002;80:213-29.

3 Gaunt N. Practical approaches to creating a security culture. Int J Med Inf 2000;60:151-7.

4 Data Protection Act, 1998.

5 Access to health records, 1990.

6 Computer Misuse Act, 1990.

7 Human Rights Act, 1998.

8 Anderson R. Undermining data privacy in health information. BN 2001;322:442-3.

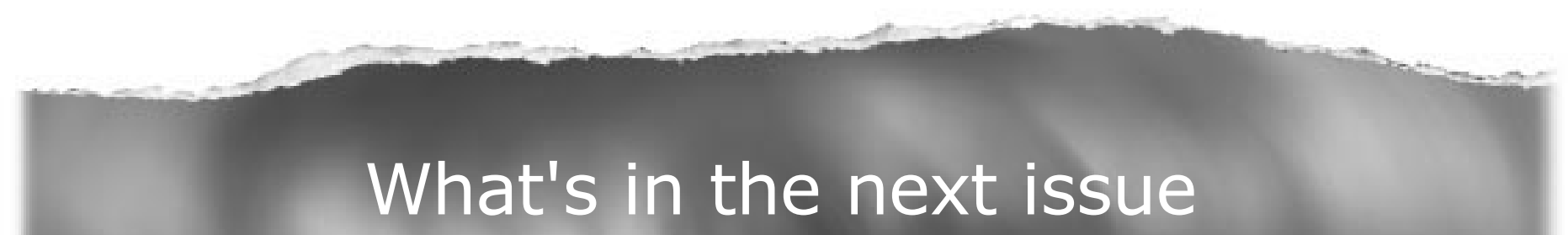

\section{Future content}

See which articles have just been accepted for publication and preview the table of contents for the next issue a month before it is published

www.jclinpath.com 